NOTICE: This is the author's version of a work that was accepted for publication in Applied Surface Science. Changes resulting from the publishing process, such as peer review, editing, corrections, structural formatting, and other quality control mechanisms may not be reflected in this document. Changes may have been made to this work since it was submitted for publication. A definitive version was subsequently published in Applied Surface Science [257, 14, 2011] DOI 10.1016/j.apsusc.2011.01.119 


\section{Accepted Manuscript}

Title: Digital image correlation approach to cracking and

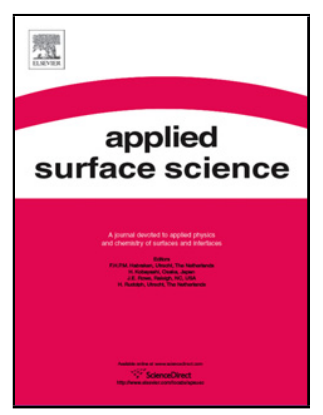

PII: S0169-4332(11)00155-3

DOI: doi:10.1016/j.apsusc.2011.01.119

Reference: APSUSC 21343

To appear in:

APSUSC

Received date:

9-12-2010

Revised date: 25-1-2011

Accepted date:

Please cite this article as: D.J. Wu, W.G. Mao, Y.C. Zhou, C. Lu, Digital image correlation approach to cracking and decohesion in a brittle coating/ductile substrate system, Applied Surface Science (2010), doi:10.1016/j.apsusc.2011.01.119

This is a PDF file of an unedited manuscript that has been accepted for publication. As a service to our customers we are providing this early version of the manuscript. The manuscript will undergo copyediting, typesetting, and review of the resulting proof before it is published in its final form. Please note that during the production process errors may be discovered which could affect the content, and all legal disclaimers that apply to the journal pertain. 
1. The DIC technique has been applied to successfully monitor and record the evolutions of full/local field strain of a thin brittle coating/ductile substrate system during tension.

2. The measurement results of stress and strain variation clarify several controversial assumptions introduced in theoretical models.

3. The fracture strength and interfacial adhesion strength of thermal barrier coatings were obtained by this method. 


\title{
Digital image correlation approach to cracking and decohesion in a brittle coating/ductile substrate system
}

\author{
D. J. Wu ${ }^{\text {a }}$, W. G. Mao ${ }^{\text {a, b }}{ }^{*}$, Y. C. Zhou ${ }^{\text {a }}$, C. $\mathrm{Lu}^{\mathrm{c}}$ \\ ${ }^{a}$ Key Laboratory of Low Dimensional Materials \& Application Technology, Ministry of Education, \\ Xiangtan University, Hunan 411105, China \\ ${ }^{\mathrm{b}}$ Aeronautical Science and Technology Key Laboratory of Aeronautical Test and Evaluation, \\ Nanchang Hangkong University, Jiangxi 330063, China \\ ${ }^{\mathrm{c}}$ Department of Mechanical Engineering, Curtin University, Western Australia 6845, Australia
}

\begin{abstract}
By using a digital image correlation technique, the full/local field strain in a brittle coating/ductile substrate system during tension has been successfully monitored. One of the most important experimental results indicates that the distribution of interfacial shear stress in the segmented coating is antisymmetric about the center, which clarifies several controversial assumptions introduced in theoretical models. Two key mechanical properties of thermal barrier coatings, fracture strength in coating and interfacial adhesion strength, were determined as $35.0 \pm 4.6$ and $14.1 \pm 3.2 \mathrm{MPa}$, respectively, which are consistent with available experimental data.
\end{abstract}

Keywords: Digital image correlation; Tensile fracture strength; Interfacial shear strength; Thermal barrier coating

\footnotetext{
* Corresponding author: W. G. Mao, Faculty of Materials, Optoelectronics and Physics, Xiangtan University, Hunan 411105, China. Tel: +86-731-58298580, Fax:+86-732-58292468, E-mail: wgmao@xtu.edu.cn.
} 


\section{Introduction}

A brittle coating bonded to ductile substrate system has been widely used due to its unique properties, such as chemical inertness, thermodynamic stability, and excellent resistance to abrasion and corrosion [1-4]. However, the premature adhesion failure of coatings subjected to mechanical load, thermal/residual stresses and temperature variation in service has limited their reliabilities and applications. Recently, various experimental methods have been developed to evaluate the fracture strength and interface adhesion properties of coatings [5-10]. In comparison with methods such as barb pullout, scratch and indentation, the tensile test is much less expensive and simpler to be performed and has also very good repeatability [6, 11-13]. Using the method, a large array of parallel cracks can be produced with a well-controlled manner on a brittle coating surface over a homogenous deformed ductile substrate. Thus, the tensile testing method can be utilized to evaluate fracture and interfacial shear strengths of a brittle coating/ductile substrate system. In the earlier investigations, different theoretical models were proposed, associated with stress distribution and coating cracking, to describe the fracture characteristic of a brittle coating/ductile substrate system. Especially, when a coating is broken into many small segmented pieces under tension, strain/stress near coating surface or interface regions is relaxed and re-distributed [11-15]. To deduce the mechanical properties of a brittle coating/ductile substrate system, it is important to accurately obtain these deformation data. However, there is not a valid experimental method and one has to resort to some disputable assumptions on the re-distribution form of strain and stress in the segmented coating. For example, the interfacial shear stress was assumed to be a sine function in a full wavelength or a quarter segment of an elliptical function [12-15]. To the 
best of our knowledge, there are few valid experimental measurements to clarify these arguments. Moreover, for deformation measurements in the cross-section of a thin coating, the conventional strain gauge methods are confronted by great difficulties due to the sample size and test temperature. It is necessary to introduce a new method to solve these strain measurement problems.

In this paper, a non-contact and non-destructive digital image correlation (DIC) technique was used to measure the full/local field strain data of a thin brittle coating/ductile substrate system. As-received thermal barrier coatings (TBCs) were chosen and studied as a typical brittle coating/ductile substrate system, consisting of ceramic coating, bond coat and substrate [1]. Full field strains in TBCs were in-situ measured during tensile tests. Based on these measurements, the mechanical properties of TBCs were obtained.

\section{Experimental procedure}

The TBC specimens were prepared by an air plasma spray technique. Here, each specimen comprises a dog-bone-shaped stainless steel substrate (SUS304) with a $10 \mathrm{~mm} \times 2$ $\mathrm{mm}$ cross-section and a $70 \mathrm{~mm}$ gauge length, a bond coat with a thickness of $100 \mu \mathrm{m}$ and a $\mathrm{ZrO}_{2}-8 \mathrm{wt} \% \mathrm{Y}_{2} \mathrm{O}_{3}$ ceramic coating with a thickness of $300 \mu \mathrm{m}$, as shown in Fig. 1. Tensile tests were conducted by a testing machine (REGER 2000) with the displacement rate control mode at a speed of $0.24 \mathrm{~mm} / \mathrm{s}$. Prior to tests, two special black and white paintings were sprayed to the polished coating surface and lateral sides by an airbrush, named as speckle patterns. The DIC technique measures displacement fields by tracking features on the specimen surface with these random speckle patterns [16]. The sampling rate was set as one image per second. The 
measuring accuracy was $\sim 0.05 \%$. Strain analysis was carried out by the commercially available Aramis 3D software. The deformation, cracking and delamination behaviors of the coating can be directly monitored by charge coupled device cameras. The test was stopped once main cracks deflected into the coating/substrate interface and resulted in decohesion. The fifteen specimens were tested at $25{ }^{\circ} \mathrm{C}$.

\section{Results and discussion}

\subsection{Full-field strain evolution in the coating surface}

A typical stress-strain curve of TBC specimens during tension is shown in Fig. 2. Several special points $A$ to $G$ in the curve are marked to reflect the cracking nucleation, initiation and propagation in coating with the increase of the external strain $\varepsilon_{s}$ in substrate, where points A to $\mathrm{D}$ denote the full-field strain evolution of the coating surface (see Fig. 1(a)), and points $\mathrm{E}$ to G represent local strain fields of the coating/substrate interface (see Fig. 1(b)). Insets in Fig. 2 are the corresponding images of strain evolution (i.e., the strain map) obtained by the DIC instrument during tests. To maintain interfacial continuities between substrate and coating, coating would have the interface shear stress due to the tensile deformation of substrate. It is seen that, from insets A to D in Fig. 2, coating undergoes the elastic deformation during the initial stage. However, as $\varepsilon_{s}$ increases, strain in the coating surface changes to the heterogeneous distribution. Several strain concentration regions appear within the monitored area and gradually propagate perpendicular to the tensile ( $x$ axis) direction. When $\varepsilon_{s}$ increases up to $0.24 \%$, three apparent strain concentration domains emerge on the surface, as indicated by $\mathrm{C} 1, \mathrm{C} 2$ and $\mathrm{C} 3$, respectively. Then, the first crack occurs in the strongest strain 
concentration region $\mathrm{C} 1$. The critical experimental data were recorded by DIC, which are used to evaluate the effective fracture strength $\sigma_{c}$ of coating with a simple formula, $\sigma_{c}=\sigma_{r}+E_{c} \varepsilon_{c}$. Here $\sigma_{r}$ and $E_{c}$ are residual axial stress and Young's modulus in the as-received coating, respectively. The average residual stress $\sigma_{r}$ was approximately $-40 \mathrm{MPa}$ predicted by our previous work $[17,18] . E_{c}$ of the as-received coating determined by nanoindentation tests was about $10 \mathrm{GPa}$ [19]. The critical axial strain $\varepsilon_{c}$ was $0.75 \% \pm 0.05 \%$ by DIC. Thus, $\sigma_{c}$ is equal to $35.0 \pm 4.6 \mathrm{MPa}$, which consists well with available experimental results $[20,21]$. With the increase of $\varepsilon_{s}$, micro-cracks at $\mathrm{C} 1, \mathrm{C} 2$ and $\mathrm{C} 3$ regions rapidly propagate throughout the width and thickness directions of coating. Then, other subsequent surface cracks gradually occur, which eventually forms an array of multiple channel cracks and segmented coatings, as shown in Fig. 3(a).

\subsection{Distribution of local strain in the segmented coating}

When these surface channel cracks arrive at the coating/substrate interface, they can not pass through the ductile substrate and generally deflect into the coating/bond coat interface, as shown in Fig. 3(b). The evolution of cracking propagation and local-field strains in the cross-sectional coating region during the failure process is shown in insets $\mathrm{E}$ to $\mathrm{G}$ in Fig. 2. It is seen that the strain heterogeneity in the coating areas is much more obvious than that in substrate, where the crack spacing, $\lambda$, is about $1.59 \mathrm{~mm}$. Since the interfacial fracture location for an air plasma sprayed as-received TBC system usually occurs within the ceramic coating close to the coating/bond coat interface due to the interfacial shear stress [1,22], we emphasize on the discussion of axial and shear strain evolution in this coating region prone to cracking 
nucleation (marked with red dashed lines in Fig. 4). It is observed from Fig. 4(a) that the maximum and minimum axial strains locate near the central and edge regions of the segmented coating, respectively. In addition, the stress-strain relationship of a brittle coating can be assumed to obey the Hooke's law before it ruptures. Therefore, these strain maps reflect the variations of stress during tests. In comparison with the theoretical strain distribution of a segmented brittle coating after tension, as schematically shown by the dash line in Fig. 4(a) [12, $13,15]$, it is seen that the recorded results by DIC are similar to that by the previous theoretical assumptions. It is interesting to note in Fig. 4(b) that the shear strain distribution in the segmented coating is almost antisymmetric in the center region, which develops gradually along the interface and reaches the maximum value near the segmented coating edges. The minimum value of shear strain occurs in the centre region of the segmented coating. The extracted three representative curves gradually shift to the higher values as $\varepsilon_{s}$ increases. Similarly, the corresponding shear stress distribution of the segmented coating has analogous characteristics due to the fracture of brittle coating. In the previous studies, the distribution of shear strain is generally assumed to be a quarter segment of an elliptical function $[12,13,15$, 23-25], as indicated by the dash dot line in Fig. 4(b). It is obvious that the increasing trend of shear strain at a given location monitored by DIC is similar to the assumptive distribution. The small difference between theoretical and experimental curves may be due to the dynamic evolution in experiments rather than the average one in the hypothetical distribution. Strain data are strongly affected by the selected locations along the coating thickness direction. Thus, the present results verify that a quarter segment of an elliptical function is more reasonable to describe the shear stress distribution of the segmented coating. Moreover, the shear strain maps 
also represent that the strongest strain concentration region takes place within coating close to the coating/bond coat interface, which is easier to induce coating delamination and spallation.

As the applied strain $\varepsilon_{s}$ continues to increase, the longer the segments break into halves, the shorter the segments remain unbroken. Eventually, the channel cracking number in coating gradually goes to a saturation state. On the other hand, when the accumulated interfacial shear stress at the coating/bond coat interface reaches the critical shear strength $\tau_{c}$ of coating, vertical channel cracks in coating gradually transform into interfacial cracks (see Fig. 3(b)). The critical shear strain $\gamma_{c}$ obtained from DIC measurements was $0.31 \% \pm 0.07 \%$ during tension. Consequently, the interfacial shear strength can be estimated with $\tau_{c}=G_{c} \gamma_{c}$, where $G_{c}=E_{c} / 2(1+v)$ is the shear modulus of ceramic coating with Poisson's ratio $v=0.1$ [17]. Here, $\tau_{c}=14.1 \pm 3.2 \mathrm{MPa}$, which agrees well with those obtained by the finite element method and shear test $[26,27]$.

\section{Conclusions}

In summary, a non-contact and non-destructive DIC technique has been successfully applied to monitor and record the evolution of full/local field strain of a thin coating system during tension. It is shown that the distributions of interfacial shear strain in the segmented coating display antisymmetric characteristic about the center, which verifies a controversial assumption in the previous theoretical models. The fracture strength of thin ceramic coating and interfacial shear strength of TBCs were determined to be $35.0 \pm 4.6$ and $14.1 \pm 3.2 \mathrm{MPa}$, respectively, which are consistent with available experimental data. The DIC technique has high reliability, non-contact and non-destructive features and can be also applied in the strain 
measurement at high temperatures.

\section{Acknowledgments}

This work has been supported by the Open Fund of Aeronautical Science and Technology Key Lab. of Aeronautical Test and Evaluation of Nanchang Hangkong University (No. HK2009005), the Fund for Young Scholars in Hunan Province (No. 08B084), the Young Teacher Fund of Ministry of Education (No. 200805301023), the Start-up Fund of Xiangtan University (No. 08QDZ07) and the National Natural Science Foundation of China (No. 10828205).

\section{References}

[1] N.P. Padture, M. Gell, E.H. Jordan, Science, 296 (2002) 280-284.

[2] M.G. Beghi, A.C. Ferrari, K.B.K. Teo, J. Robertson, C.E. Bottani, A. Libassi, B.K. Tanner, Appl. Phys. Lett., 81 (2002) 3804-3806.

[3] N. Holten-Andersen, G.E. Fantner, S. Hohlbauch, J.H. Waite, F.W. Zok, Nature Mater., 6 (2007) 669-672.

[4] C. Donnet, A. Erdemir, Surf. Coat. Technol., 180-181 (2004) 76-84.

[5] J. Yan, T. Leist, M. Bartsch, A.M. Karlsson, Acta Mater., 56 (2008) 4080-4090.

[6] A.P. McGuigan, B.D. Huey, G.A.D. Briggs, O.V. Kolosov, Y. Tsukahara, M. Yanaka, Appl. Phys. Lett., 80 (2002) 1180-1182.

[7] H. Hirakata, M. Kitazawa, T. Kitamura, Acta Mater., 54 (2006) 89-97.

[8] S.Q. Guo, D.R. Mumm, A.M. Karlsson, Y. Kagawa, Scripta Mater., 53 (2005) 1043-1048. 
[9] O. Borrero-López, M. Hoffman, A. Bendavid, P.J. Martin, Thin Solid Films, 518 (2010) 4911-4917.

[10] O. Borrero-López, M. Hoffman, A. Bendavid, P.J. Martin, Acta Mater., 56 (2008) $1633-1641$.

[11] X.C. Zhang, B.S. Xu, F.Z. Xuan, S.T. Tu, J. Appl. Phys., 103 (2008) 023519.

[12] C. Xie, W. Tong, Acta Mater., 53 (2005) 477-485.

[13] B.F. Chen, J. Hwang, I.F. Chen, G.P. Yu, J.H. Huang, Surf. Coat. Technol., 126 (2000) 91-95.

[14] D.C. Agrawal, R. Raj, Acta Metall., 37 (1989) 1265-1270.

[15] J.H. Jeong, D. Kwon, J. Adhes. Sci. Technol., 12 (1998) 29-46.

[16] C. Efstathiou, H. Sehitoglu, J. Carroll, J. Lambros, H.J. Maier, Acta Mater., 56 (2008) 3791-3799.

[17] W.G. Mao, Y.C. Zhou, L. Yang, X.H. Yu, Mech. Mater., 38 (2006) 1118-1127.

[18] Q. Chen, W.G. Mao, Y.C. Zhou, C. Lu, Appl. Surf. Sci., 256 (2010) 7311-7315.

[19] W.G. Mao, Q. Chen, C.Y. Dai, L. Yang, Y.C. Zhou, C. Lu, Surf. Coat. Technol., 204 (2010) $3573-3577$

[20] Y. Yamazaki, A. Schmidt, A. Scholz, Surf. Coat. Technol., 201 (2006) 744-754.

[21] D. Schwingel, R. Taylor, T. Haubold, J. Wigren, C. Gualco, Surf. Coat. Technol., 108-109 (1998) 99-106.

[22] M. Gell, J. Eric, V. Krishnakumar, K. McCarron, B. Barber, Y.H. Sohn, V.K. Tolpygo, Surf. Coat. Technol., 120-121 (1999) 53-60.

[23] A.P. McGuigan, G.A.D. Briggs, V.M. Burlakov, M. Yanaka, Y. Tsukahara, Thin Solid 
Films, 424 (2003) 219-223.

[24] F. Ahmed, K. Durst, S. Rosiwal, J. Fandrey, J. Schaufler, M. Goken, Surf. Coat. Technol., 204 (2009) 1022-1025.

[25] B.E. Alaca, M.T.A. Saif, H. Sehitoglu, Acta Mater., 50 (2002) 1197-1209.

[26] P.F. Zhao, C.A. Sun, X.Y. Zhu, F.L. Shang, C.J. Li, Surf. Coat. Technol., 204 (2010) 4066-4074.

[27] T.A. Cruse, R.C. Dommarco, P.C. Bastias, J. Eng. Mater. Technol., 120 (1998) 26-32. 


\section{Figure captions}

Fig. 1. A specimen with speckle patterns, where the green regions indicate the monitored areas during tensile tests. (a) the surface coating region, (b) the cross-sectional coating region.

Fig. 2. A typical stress-strain curve of TBCs during tensile tests. Insets are strain maps that show the heterogeneity of full strain field and cracking formation in the coating. The different phases of cracking nucleation, propagation and interface delamination in coating are marked by points A to $\mathrm{G}$ along the curve, where A: $\varepsilon_{s}=0.05 \%$, B: $\varepsilon_{s}=$ $0.10 \%, \mathrm{C}: \varepsilon_{s}=0.24 \%, \mathrm{D}: \varepsilon_{s}=0.37 \%, \mathrm{E}: \varepsilon_{s}=0.52 \%, \mathrm{~F}: \varepsilon_{s}=0.75 \%$, and G: $\varepsilon_{s}=$ $0.93 \%$. Eventually, a segmented coating with crack spacing $\lambda$ forms.

Fig. 3. Scanning electron microscope observations of TBCs after tensile failure: (a) the typical channel cracking morphology on the coating surface and (b) the cracking propagation near the coating interface. The cracking features are consistent well with the alternated red and blue regions by DIC, as shown in Fig. 2.

Fig. 4. The evolution of axial strain (a) and shear strain (b) in the cross-section of a segmented coating close to the coating/substrate interface during tensile tests. The dash and dash dot lines show, respectively, the distributions of hypothetic normal and shear strains of a segmented coating used in the previous works. 


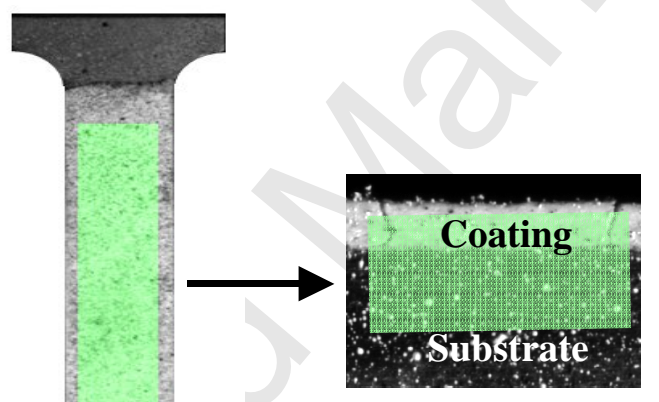

(b)

(a)

Fig. 1 


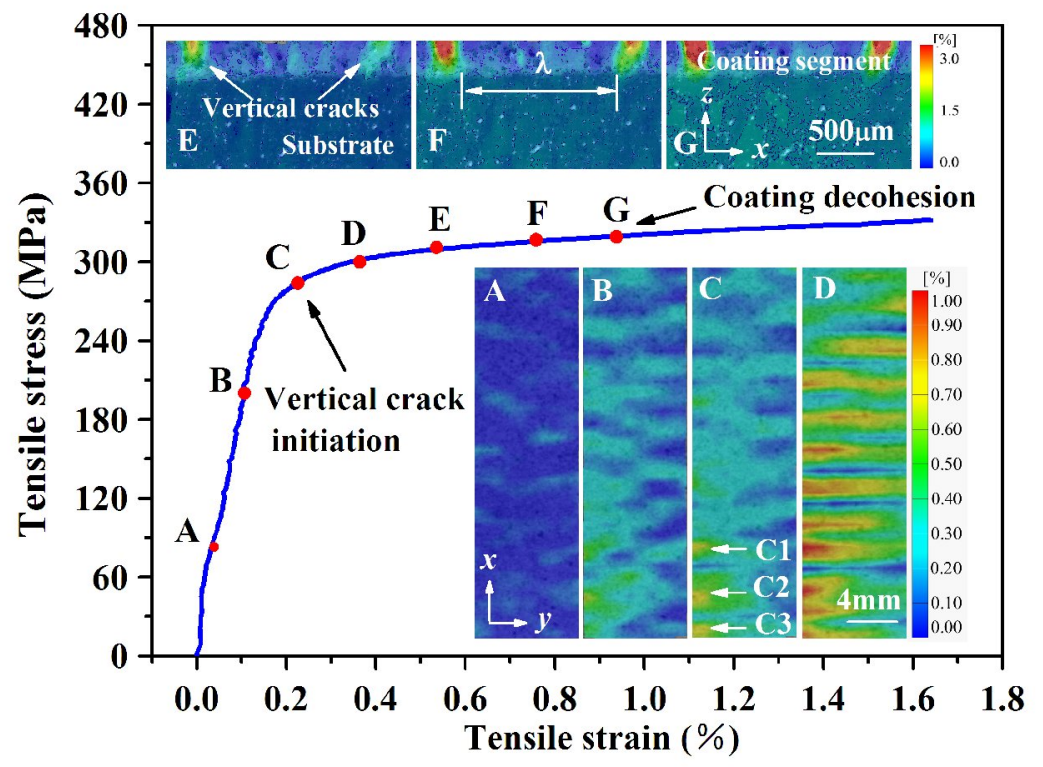

Fig. 2 

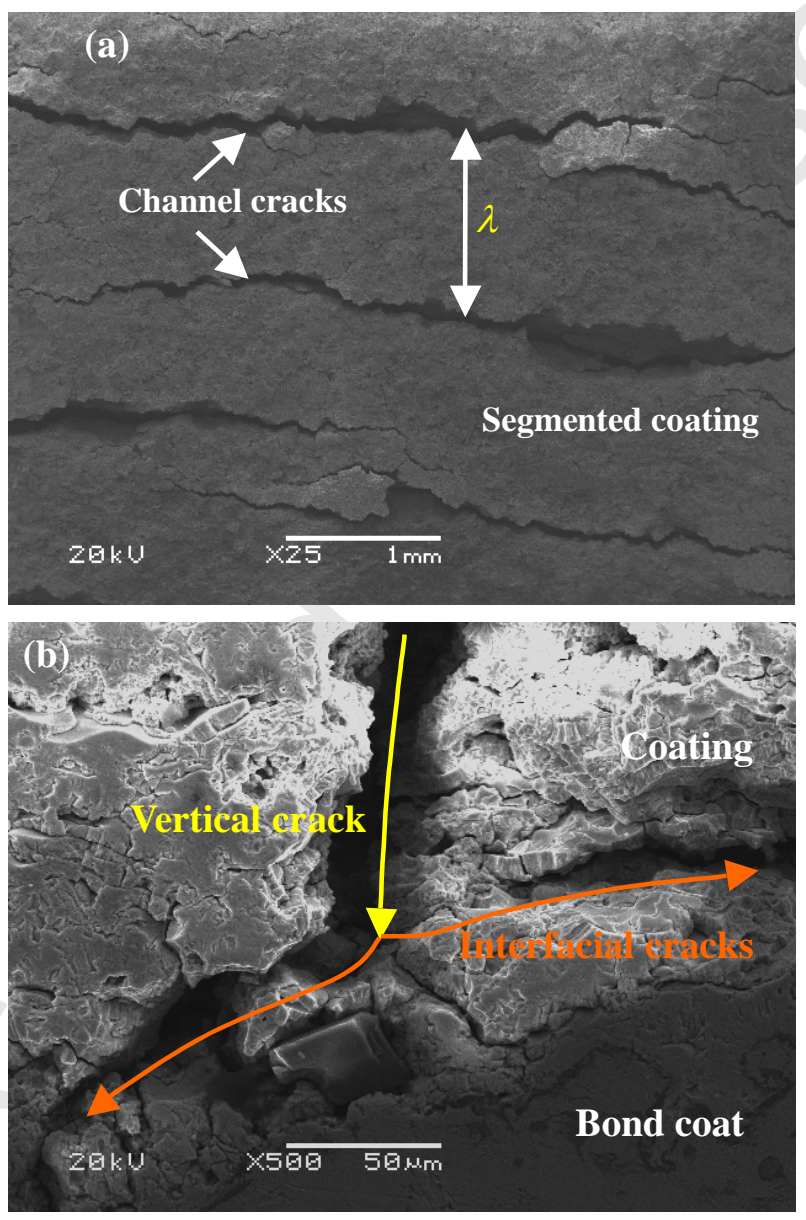

Fig. 3 


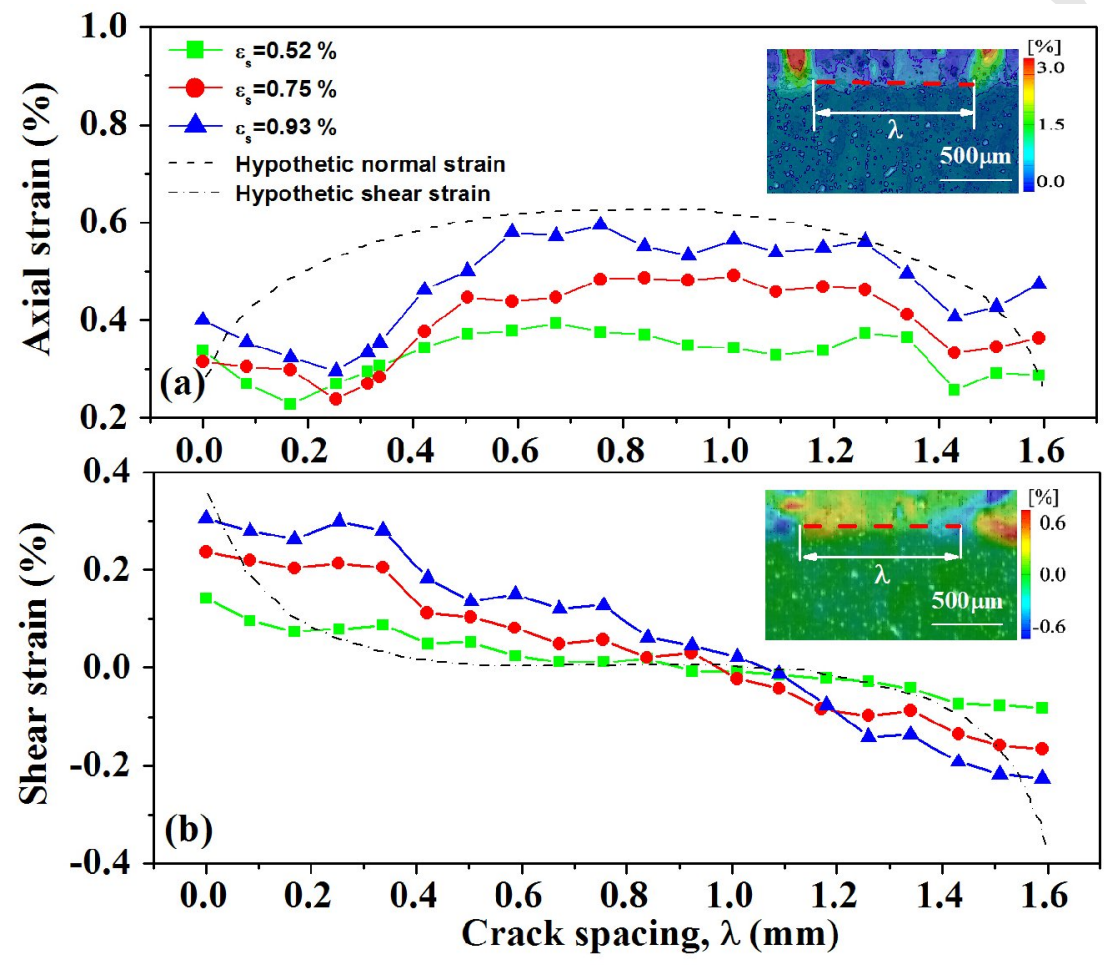

Fig. 4 